\title{
EL RECONOCIMIENTO COMO PRESUPUESTO DISCURSIVO PARA UNA CONCEPCIÓN PLURIVERSALISTA DE DERECHOS HUMANOS
}

\author{
Anderson Vichinkeski Teixeira*
}

Fecha de recibido: 29 de diciembre de 2012

Fecha de aprobado: 6 de marzo de 2013

Artículo de reflexión

Forma de citación: Vichinkeski, A. (2013). El reconocimiento como presupuesto discursivo para una concepción pluriversalista de Derechos Humanos. Revista Prolegómenos. Derechos y Valores, 16, 31, 117-130.

\section{Resumen}

En este artículo se analizan los fundamentos filosóficos de la doctrina de los derechos humanos a partir de la Teoría Crítica del Reconocimiento. En un primer momento, se recordarán los orígenes de los conceptos de persona humana y dignidad humana, según las contribuciones de la tradición judaico-cristiana, buscando demostrar la vinculación entre la concepción de derechos humanos y una tradición cultural específica. Posteriormente, intentamos introducir la propuesta teórica de reinterpretación de los derechos humanos con base en postulados discursivos, antes postulados normativos, con fines de aproximar las partes implicadas en el discurso de acuerdo con las tradiciones culturales y demás vínculos capaces de producir relaciones de reconocimiento. Finalmente, se analiza cómo la Teoría del Reconocimiento puede colaborar para una redefinición epistemológica de la categoría de los derechos humanos.

\section{Palabras clave}

Derechos humanos, Rreconocimiento, Pluriuniversalidad

\section{RECOGNITION AS A DISCURSIVE POSTULATE FOR A PLURIVERSALIST CONCEPTION OF HUMAN RIGHTS}

\begin{abstract}
This article analyzes the philosophical foundations of human rights doctrine from the perspective of the Critical Theory of Recognition. Initially, the article revisit the origins of the concepts of human person and human dignity according to the contributions from the Jewish-Christian tradition, aiming to show the bonds between the conception of human

* Doctor (2009) en Teoría y Historia del Derecho por la Università degli Studi di Firenze (IT), con periodo de investigación doctoral en la Facultad de Filosofía de la Université Paris Descartes-Sorbonne. Periodo de investigación posdoctoral en la Università degli Studi di Firenze (2010). Maestría (2005) en Derecho del Estado (PUC/RS). Profesor del Programa de Postgrado en Derecho de la Universidade do Vale do Rio dos Sinos (UNISINOS). Consultor jurídico y abogado. Otros escritos del autor están disponibles en www.andersonteixeira.com
\end{abstract}


rights and a specific cultural tradition. Afterwards, the article introduces the theoretical proposal of reinterpretation of human rights based on discursive postulates, before normative postulates, with the purpose to bring closer the parties involved in the debate according to the cultural traditions and other bonds capable to produce relations of recognition. Finally, the article analyzes how the Theory of Recognition could collaborate with an epistemological redefinition of the category of human rights.

\title{
Keywords
}

Human Rights, Recognition, Pluri universality

\section{O RECONHECIMENTO COMO PRESSUPOSTO DISCURSIVO PARA UMA CONCEPÇÃO PLURIVERSALISTA DOS DIREITOS HUMANOS}

\begin{abstract}
Resumo
Neste artigo discutem-se os fundamentos filosóficos da doutrina dos direitos humanos a partir da Teoria Crítica do reconhecimento. Em primeiro lugar, lembramos as origens dos conceitos da pessoa humana e à dignidade humana, de acordo com as contribuições da tradição judaico-cristã, procurando demonstrar a ligação entre a concepção de direitos humanos e uma tradição cultural especifica. Depois, tentamos apresentar a proposta teórica de reinterpretação dos direitos humanos com base em postulados discursivos, antes postulados normativos, para fins de aproximar as partes envolvidas no discurso de acordo com as tradições culturais e outras semelhanças existentes capazes de produzir relações de reconhecimento. Finalmente, analisa-se como a teoria do reconhecimento pode trabalhar para redefinição epistemológica da categoria dos direitos humanos.
\end{abstract}

\section{Palavras-chave}

Direitos Humanos, Reconhecimento, Pluri universalidade

\section{INTRODUCCIÓN}

En contextos socio-políticos donde las principales normas definidoras de derechos humanos ya fueron constitucionalizadas el debate sobre la legitimidad de tales normas no suele ser un problema muy significativo, pues el hecho de existir un consenso minino, suficiente para convertirlas en normas fundamentales de orden constitucional, termina centrando el foco del debate en el ámbito de la eficacia de los derechos fundamentales. Pero la realidad es otra cuando existen diferentes culturas procurando la validez de sus respectivos principios, aunque muchos de los axiomas establecidos por esas culturas sean substancialmente opuestos entre sí, o sea, cuando reconocer la validad de una formación cultural significa negar la validad de otra.

Tal realidad es lo que ha enfrentado la casi totalidad de los países europeos: tornar compatibles, bajo una perspectiva multicultural, diferentes formaciones culturales que refutan la propia diversidad cultural en sí. Sea en el ámbito nacional o en el de la Unión Europea, el desafío de la convivencia multicultural se constituye hoy en uno de los mayores desafíos políticos y jurídicos en Europa. ¿Cómo hacer para que un occidental, oriundo de la tradición judaico-cristiana, acepte la legitimidad del uso de la burka por parte de mujeres musulmanas o los supuestos beneficios so- 
ciales e infectológicos traídos por la infibulación de jóvenes adolescentes? ¿Cómo hacer para que un árabe, oriundo de la tradición islámica, reconozca la legitimidad moral - y por consecuencia jurídica - del matrimonio entre, por ejemplo, dos hombres holandeses que pretenden adoptar hijos?

Analizando solamente la perspectiva de cada tradición cultural, la aceptación de tales conductas sería una negación de principios fundamentales para las respectivas tradiciones. Sin embargo, el problema es agravado cuando pensamos en cómo un Estado nacional europeo, constituido a partir de la tradición judaico-cristiana, debe posicionarse en las situaciones en que es llamado a dar reconocimiento a derechos culturales pertenecientes a tradiciones que niegan la legitimidad cultural de aquel a quien están pleiteando el reconocimiento de tales derechos culturales. Esa paradoja aparente -que es, en efecto, una paradoja real- hace parte de la realidad hodierna de alemanes, italianos, franceses, entre otros pueblos europeos que están viendo formarse en su interior algo que podríamos llamar "para-naciones", es decir, contextos sociales dotados de simbología, lengua y vínculos intersubjetivos propios, autónomos en relación a los del Estado nacional hospedero; verdaderas naciones paralelas, las cuales ya están yendo para nuevas generaciones de descendientes, pues el fenómeno migratorio de mayor expresión empezó hace más de 50 años.

Si desde el punto de vista de cada tradición cultural la cuestión se muestra insoluble, a partir de la doctrina de los derechos humanos eso podría ser fácilmente solucionado: nada que no reconozca la legitimidad universal de los derechos consagrados en las declaraciones universales de derechos, en la Carta de las Naciones Unidas y en las principales normas internacionales definidoras de derechos humanos podrá ser visto como legítimo. En suma, bastaría declarar la ilegitimidad de las conductas específicas y aceptar solamente lo que resta de la tradición en objeto - eso cuando reste algo.

Tal respuesta autista y simplista ha sido ciegamente profesada por los defensores irrestrictos de la universalidad de los derechos humanos, entre los cuales se destacan los juristas, principalmente estadounidenses, que apoyaron la posición del gobierno Bush Jr. en sus invasiones a Afganistán e Irak. La historia reciente probó que la negación extrema de la existencia del otro, del diferente, no tiene en la solución bélica su mejor alternativa. Los misiles de los EUA y de sus aliados colaboraron para aumentar la instabilidad entre las tradiciones culturales conflictivas, en lugar de cohibir futuros enfrentamientos, sea de proporción pequeña o grande.

Lo que pretendemos en las próximas páginas es discutir sobre la posibilidad de una reinterpretación de la doctrina de los derechos humanos a partir de la Teoría Crítica del Reconocimiento. En otro momento fue tratado el tema de los fundamentos de la orden internacional bajo la óptica de una alternativa al universalismo de la doctrina de los derechos humanos: una concepción pluriversalista de la orden internacional que favorezca el fortalecimiento de instancias (comunidades) regionales de Estados-naciones, de modo que la conflictividad sea reducida mediante, por un lado, la profundización de los vínculos intersubjetivos que internamente sustentan las tradiciones culturales $y$, por otro, la aproximación de esas tradiciones en el debate sobre el contenido substancial de los derechos que gozan de reconocimiento por todos los implicados en el debate (Teixeira, 2011). Sin embargo, en aquel texto monográfico el análisis se concentró en los aspectos procedimentales relativos a lo que sería un orden internacional pluriversalista, dejando de entrar en el debate sobre el contenido substancial de los derechos humanos.

En el presente artículo retomaremos, brevemente, los principales caracteres atinentes a 
la fundamentación ético-teológica de la doctrina de los derechos humanos, de modo que podamos proponer una desconstrucción, a partir de la Teoría Crítica del Reconocimiento, de la supuesta normatividad universal de esa doctrina. Al final, defenderemos que el reconocimiento puede constituirse en un presupuesto discursivo de los derechos humanos, pues la complejidad de las diversas instancias de normatividad jurídica tiene en las especies de reconocimiento sus instrumentos más adecuados para la necesaria producción de reflexividad entre el yo y el otro.

\section{LA FUNDAMENTACIÓN ÉTICO-TEOLÓGICA DE LA DOCTRINA DE LOS DERECHOS HUMANOS}

Parece algo incuestionable la vinculación del surgimiento de la doctrina de los derechos humanos al iusnaturalismo y, por consecuencia, a la tradición judaico-cristiana. Para fines argumentativos, recuperaremos solamente dos legados evidentemente cristianos que se han constituido en elementos centrales para la doctrina de los derechos humanos: el concepto de persona humana y el principio de dignidad humana.

En lo que tiene que ver con el concepto de persona humana, es notorio ver que tal noción fue producida en el Occidente, pues, de San Agustín a Tomás de Aquino la figura central en el mundo era el individuo comprendido como persona, en vez de súbdito, siervo o cualquier otra categoría conceptual que le retirase su condición de individualidad humana. ${ }^{1}$ Existiría solamente la sumisión a Dios y a los preceptos de la Iglesia. El pensamento de Hannah Arendt se hace muy claro en ese sentido cuando afirma que a própria vida é sagrada, mais sagrada que tudo mais no mundo; e o homem é o ser supremo sobre a terra (Arendt, 1972, p. 83). En otra obra, la autora

\footnotetext{
Sobre las vinculaciones históricas entre la doctrina del derecho natural, predecesora de los derechos humanos, y la tradición judaico-cristiana, recomendamos Strauss (1986).
}

asocia el cristianismo a la consolidación de la vida como bien supremo:

O motivo pelo qual a vida se afirmou como ponto último de referência na era moderna e permaneceu como bem supremo para a sociedade foi a moderna inversão de posições que ocorreu dentro da textura de uma sociedade cristã, cuja crença fundamental nos sacros santidade da vida sobrevivera à secularização e ao declínio geral da fé cristã, que nem mesmo chegaram a abalá-la. Em outras palavras, a moderna inversão imitou, sem questionar, a mais significativa viravolta com a qual o cristianismo irrompera no cenário do mundo antigo, viravolta politicamente mais importante e, pelo menos historicamente, mais duradoura que qualquer conteúdo dogmático ou crença específica. Pois a "boa nova" cristã da imortalidade da vida humana individual invertera a antiga relação entre o homem e o mundo, promovendo aquilo que era mais mortal, a vida humana, à posição de imortalidade ocupada até então pelo cosmo (Arendt, 2002, p. 327).

Arendt es precisa al destacar que la secularización del poder político no representó la fragilización de la importancia de la noción de persona humana, sino su fortalecimiento. En la modernidad fue esa concepción de hombre que tornó posible cualquier intento - especialmente después del Holocausto - de cambiarse el paradigma axiológico que balizaba la actividad estatal, pasando de un Estado cuyo fin supremo era su propio bien para un Estado cuyo bien mayor es la vida humana.

El principio de la dignidad humana no consiste tan solo en el hecho de ser ésta, diferente de otras cosas, un ser considerado en sí mismo, con el fin en sí propio y nunca un medio para la consecución de determinado objeto; es también consecuencia de la voluntad racional del hombre, pues solo la persona tiene autonomía suficiente para poder guiarse por leyes que ella 
misma elabora. ${ }^{2}$ Por eso, al ser humano fue atribuida la dignidad, en vez de un precio, como lo que se vincula a las cosas. La dignidad de la persona resulta del hecho de ser el humano el punto inicial de donde surgen todos los derechos. La individualista "Era de los derechos" referida por Bobbio construye sus estructuras normativas más fundamentales a partir del principio de la dignidad humana (1997, p. 59).

Corolario inmediato del principio de dignidad humana es, por cierto, el ideal de humanidad, o sea, la unidad del género humano. En consecuencia, de la sacralización de la vida y de su necesidad de protección resultan lo que el abad de Saint-Pierre y Immanuel Kant llamaban de "paz perpetua", algo que se tornó, en el siglo XX, mucho más que un ideal: un bien a ser buscado por todos los pueblos; un bien que toda humanidad debería siempre tutelar. Fue la búsqueda por ese bien que posibilitó a la doctrina de los derechos humanos llegar en el siglo XXI como una gran ideología dentro de la cual todas las corrientes iusfilosóficas deberían encuadrarse, restándoles apenas la tarea de discutir los medios para tornar efectivos los principios, sin dar mucha relevancia a la discusión sobre la legitimidad y validad de tales principios supuestamente universales.

2 El ideal kantiano de dignidad de la persona humana ilustra muy bien eso: "Se, pois, existirem um princípio prático supremo e um imperativo categórico no que diz respeito à vontade humana, deverão ser tais que, da representação daquilo que é necessariamente um fim para todos porque é fim em si mesmo, constitua um princípio objetivo da vontade, que possa, por conseguinte, servir de lei prática universal. O fundamento deste princípio é: a natureza racional existe como fim em si. É assim que o homem se representa necessariamente a sua própria existência; e neste sentido, esse princípio é um princípio subjetivo das ações humanas. Mas é também assim que qualquer outro ser racional se representa a sua existência, em conseqüência do mesmo fundamento racional válido para mim; é pois, ao mesmo tempo, um princípio objetivo, do qual, como princípio prático supremo, hão de se poder derivar todas as leis da vontade. O imperativo prático será, pois, o seguinte: age de tal maneira que possas usar a humanidade, tanto em tua pessoa como na pessoa de qualquer outro, sempre e simultaneamente como fim e nunca simplesmente como meio" (Kant, 2003, p. 59).
Sin embargo, las guerras humanitarias, la creciente desigualdad entre los Estados pobres y las grandes potencias, la derrocada moral de instituciones internacionales como las Naciones Unidas, después de la segunda guerra contra el Irak, además de la condición de inseguridad colectiva que pasó a vigorar en las relaciones internacionales desde los atentados del 11 de septiembre de 2001, nos permite cuestionar si realmente existe una verdadera humanidad como, supuestamente, debería ser. En este contexto, somos forzados a reconocer la actualidad del pensamiento de Carl Schmitt cuando afirmaba que la humanidad è uno strumento ideologico particolarmente idoneo alle espansioni imperaliste ed è, nella sua forma etico-umanitaria, un veicolo specifico dell'imperialismo economico (Schmitt, 1972, p. 139).

Defender la humanidad, entendida como un concepto absoluto, homogéneo y genérico, ha sido algo muy útil al Estado que pretende apropiarse de este concepto y utilizarlo como le sea más conveniente, en vez de ser considerada como una causa para la formación de una comunidad de destino universal entre pueblos que defienden la ubicuidad de la "paz perpetua". Aún recordando Schmitt, vemos que chi parla di umanità, vuol trarvi in inganno (1972, p. 139).

Definiendo la doctrina de los derechos humanos como una ideología política, estamos apenas haciendo eco a lo que fue dicho por uno de los mayores exponentes de esa doctrina en el siglo XX: Richard Falk. En el período de la bipolarización política que dividió el mundo durante la Guerra Fría, Falk sostenía que to promote human rights necessarily implies mounting an anticommunist ideological attack, with all that this portends for deteriorating Soviet-American relations (Falk, 1981, p. 25). Así el autor, indirectamente, demostraba que la doctrina de los derechos humanos es substancialmente una ideología política, pues reconoce que la esencia de la protección de los derechos humanos es producto de una confrontación entre fuerzas sociales opuestas y no puede ser entendida como una actividad 
creadora de derechos o como una forma de persuasión racional (Falk, 1981, p. 34).

Si fuera correcta la concepción iusnaturalista de que algunos derechos son inherentes a la naturaleza humana y que, simplemente por eso, deben ser respetados por cualquier sociedad civilizada, no ocurriría lo que, según Falk, es la principal dificultad de la lógica naturalista: The main difficulty with naturalist logic relates to the vagueness of norms and ambiguity of the mandate (Falk, 1981, p. 43).

La vaguedad de la prescripción normativa, a la cual refiere Falk, caracteriza la retórica de los derechos humanos, una vez que es el resultado directo de la pretensión universal que esta doctrina se propone alcanzar. La indiferencia por el particular, por las realidades propias de las comunidades regionales y nacionales, sumada a la necesidad de que el concepto tiene que restringir en su propia existencia lingüística toda la dimensión fenomenológica presentada por los más variados pueblos, etnias y culturas, hace que el contenido de los derechos humanos falle en efectividad, bien sea por la vaguedad o por la demasiada precisión normativa.

El pensamiento de Falk ilustra muy bien como la universalidad de la doctrina de los derechos humanos también debería constituirse en principio discursivo. Él critica el carácter autoritario de muchas formulaciones existentes en el derecho internacional sobre derechos humanos, reconociendo incluso que ésta es una doctrina peculiar al liberalismo occidental que busca ser aplicada como si fuera universal (Falk, 1981, p. 137). Falk además añade que reformulaciones en lo que se refiere al contenido y a las prescripciones dadas por tal doctrina necesitan ser tratadas dentro de un contexto donde los países de las más diversas ideologías sean oídos, pero considera que, no obstante, las críticas existentes, la Declaración Universal de los Derechos del Hombre y del Ciudadano es un documento que se encuadra en los intereses de países no sólo capitalistas y occidentales (Falk, 1981, p. 138). Según él, es como si todo el debate doc- trinario existente en torno de la propia validad universal de muchos de los derechos presentes en tal documento fuera mero debate sobre la efectividad o instrumentalización de preceptos normativos existentes e incontestados.

Como forma de perfeccionamiento del contenido del catálogo de los derechos humanos, el autor entiende que es dentro de un contexto de global governance solidamente fundado en un proyecto normativo, capaz de contar sobre todo con una World Court, que será posible que sean rotas las lógicas autoritaristas y pretorianas que preponderan en los países que no reconocen o no han implementado la doctrina de los derechos humanos (Falk, 1981, pp. 181-182; Id., 1995, pp. 249-250; Id., 1999, pp. 182-184). En suma, el autor propone que se establezca un debate intercultural -inclusive llamando ideologías distintas- sobre el contenido material de los derechos humanos, pero a priori ya establece los principios del discurso racional, las posibilidades de elección que nosotros tendremos y cuales objetivos el debate deberá alcanzar (Teixeira, 2008, pp. 212-213).

\section{DECONSTRUYENDOLAABSTRACCIÓN DE LA NORMATIVIDAD UNIVERSAL DE LOS DERECHOS HUMANOS A PARTIR DE LA TEORÍA CRÍTICA DEL RECONOCIMIENTO DE AXEL HONNETH}

Un punto de partida para cualquier análisis teórico de la doctrina de los derechos humanos está en reconocer el hecho de que ella fue construida con base en la noción de reconocimiento-reciprocidad: la universalidad del género humano sería condición suficiente para permitir que los elementos definidores de la noción de ego (yo, self) fuesen constituidos, necesariamente, a partir de los mismos elementos que caracterizan el alter (el otro, el diferente). En otras palabras, mi propia idea de yo solamente puede ser construida en una relación reflexivo-identificante con individuos que se guían por los mismos principios de la razón práctica que conducen mi conducta, 
pues el reconocimiento-reciprocidad convierte los principios racionales compartidos en verdaderos axiomas que no pueden ser contestados por aquellos agentes racionales que hacen parte de determinada comunidad ética.

Sin embargo, como la doctrina de los derechos humanos presupone la universalidad de sus principios racionales, tales axiomas deberían valer incontestablemente para todo el género humano, transformando en una única comunidad ética la humanidad como un todo. Lo que debemos cuestionar en ese momento es la necesidad y utilidad de recorrer, desde el punto de vista discursivo, solamente al reconocimiento-reciprocidad para fundamentar la construcción intersubjetiva de los principios básicos sobre los cuales están fundados los derechos humanos. La historia de la teoría del reconocimiento, desde Hegel, pasando especialmente por Paul Ricoeur, Georg Mead, Maurice Merleau-Ponty, hasta llegar a Axel Honneth, posee especificidades y desarrollos en condiciones de establecer nuevos referenciales epistemológicos para los derechos humanos.

Autores como Charles Taylor han manifestado que el principio de reciprocidad pertenece a lo que sería una "política de reconocimiento", que tendría el principio de la igualdad universal (o principio de la igual dignidad) como uno de sus principios-guía (Taylor, 2005, p. 09-62). ${ }^{3}$ Según Taylor, el principio de la igual dignidad se basa en una potencialidad humana universal que torna todos los seres humanos igualmente dignos de respeto, independientemente de las culturas en las que se encuentren inseridos (2005, pp. 27-28).

Sin embargo, el principio de la igual dignidad, defendido en ese mismo sentido por Taylor, se pondrá como absolutamente adverso a las diferencias individuales y culturales: se trata de un principio que creará una idea abstracta del ser humano sin llevar en consideración lo que ocurre dentro de las realidades factuales a las cua-

3 Ese texto fue publicado originalmente en Taylor, C. (1992). The Politics of Recognition. Princeton: Princeton University Press. les los individuos pertenecen (Zarka, 2007, pp. 42-43). Se trata de una actitud homogeneizante que busca anular todas las diferencias intraculturales y la propia diversidad intercultural en nombre de una identidad universal $y$, por consecuencia, trascendental - la cual será, en general, de matriz kantiana. El individuo titular de los derechos decurrentes de esa dignidad sería una mera abstracción amorfa y descontextualizada en relación con cualquier tradición cultural, una vez que, incluso dentro de Occidente, la progresiva fragmentación en subculturas y microculturas -fenómeno cada vez más profundo y veloz- hace que sea inocuo hablar de un "ser humano universal". Una postura homogeneizante en forma de mundialización está lejos de hacer desaparecer las diferencias entre las identidades colectivas, pues, al contrario, termina por atestar la irréductible ténacité (Zarka, 2007, p. 37) que ellas poseen. Como prueba de eso, basta observar los constantes conflictos interculturales que comprueban la imposibilidad de cualquier reductio ad unum entre las diversas culturas, ideologías y religiones que se enfrentan, sea en el escenario internacional o en el ámbito interno de los países.

Si reconocemos que fenómenos como el multiculturalismo y los diversos procesos de globalización fueron suficientemente fuertes para exponer las fragilidades argumentativas de los presupuestos considerados como universales de los derechos humanos, concluiremos que la justificación racional del sistema jurídico-normativo necesita aproximarse al mismo ritmo de complejización de las relaciones sociales, políticas y económicas. El yo que reconoce el otro realiza un acto que está vinculado a los caracteres más pormenorizados de aquella relación subjetiva en sí. El yo como institución, el yo como Estado nacional, el yo como amigo y el yo como amante, no comparten la misma forma de reconocimiento, pues éste tiene diversas especies propias para los muchos contextos intersubjetivos - aunque en el caso de la doctrina de los derechos humanos el reconocimiento-reciprocidad sea visto por sus paladines como plenamente satisfactorio en todas las situaciones. 
Para una mejor comprensión de las diferentes especies que la historia de la teoría del reconocimiento atribuye a este concepto, recordemos brevemente sus cuatro concepciones tradicionales más comúnmente referidas:

(1) Reconocimiento-identificación: empiezacon el acto de identificar, de reconocer una cosa como ella misma. Pero es más que la identificación de una cosa: es la identificación de sí mismo en aquella cosa. El reconocimiento-identificación se asemeja al reconocimiento-reciprocidad y al reconocimiento mutuo a medida que el yo necesita del otro para descubrir quién es. En estadios más avanzados, la identificación pasa por el nivel cultural y político, pero conservando siempre el mismo modelo de construcción de identidad entre el yo y el otro. La alteridad surge, primordialmente, a partir de esa forma de reconocimiento.

(2) Reconocimiento-atestación: es un ato formal y positivo, es decir, mediante prueba incontestada reconocida por todos, como un título universitario, documento de identidad o el derecho subjetivo de alguien, por ejemplo. Es el reconocimiento social (muchas veces institucional) de la capacidad individual o que un individuo pertenece a una determinada categoría. Sin embargo, lo que se considera no es propiamente la capacidad del individuo para consigo mismo: en el reconocimiento-atestación la capacidad social es el determinante de lo que el individuo puede ofrecer a la sociedad.

(3) Reconocimiento-responsabilidad: es una forma de reconocimiento que tiene doble sentido: moral y jurídico. Se vincula directamente al reconocimiento-identificación, pues el individuo es responsable solamente por los actos a los cuales haya, de forma conciente, dado causa y que le sea posible reconocer como suyos, en otras palabras, como consecuencia de su identidad individual, originado a partir de una acción pasible de ser situada en el precurso de la vida del individuo. Desde el punto de vista jurídico, restringe la dimensión de la cuestión de la responsabilidad solamente a la responsabilidad subjetiva. La conciencia sobre la propia responsabilidad del yo es algo construido con base en esa forma de reconocimiento.

(4) Reconocimiento-reconciliación: empieza durante un conflicto y se consolida después de su término. Es un reconocimiento terminal que superó un equívoco en el conocimiento entre las partes, de modo que el resultado final será la superación de las diferencias en una nueva realidad cognitiva en que cada individuo implicado en el conflicto anterior pasa a pensar su identidad individual como correspondiente a la identidad individual del otro. Ejemplos clásicos de conflictos de esa naturaleza son vistos en la relación entre servidor y señor, entre amigo y enemigo, pues el yo no podría ser entendido de tal forma -como servidor o enemigo, por ejemplo- caso en el que no existiera el otro para determinar quién soy yo; en contrapartida, así como el yo es definido inversamente por el otro, yo también sirvo para definir el otro como negación de mi yo. Encerrado el conflicto, las identidades implicadas pasan a tener en el otro la posibilidad de la descubierta del yo que otrora escapaba a sus propios ojos y comprensión. ${ }^{4}$

Se resalta que la palabra reconocimiento tiene origen en un verbo dotado de voz activa $y$ voz pasiva: reconocer y ser reconocido. Reconocer es un acto unilateral que exprime la pretensión de ejercer una superioridad intelectual en el campo de las aserciones significativas en relación a quién debe ser reconocido (Ricoeur, 2003, pp. 39-40). Por eso, para que el reconocimiento pueda crear identidad recíproca, especialmente en el plano político, se hace necesario establecer una relación dialéctica entre las partes, es decir, un reconocimiento mutuo. Reconocer una cosa como ella misma, como idéntica al yo que se encuentra en el otro y no

4 Para ulteriores estudios sobre el tema del reconocimiento, ver: Ricoeur (2003 y 1990), Zarka (2007, 2000 y 2002) Torodov (1989), Honneth (2000, 2006 y 2007, Ferry (1991 y 2001) Benoist (2005) y Hall (1979). 
como cualquier otra cosa que no sea ella misma, implica en distinguirla de todas las demás (Ricoeur, 2003, p. 42).

De la misma forma que mi yo se encontró en el otro la reciprocidad de la relación hace con que el otro se encuentre en mí. Pero por tratarse de un proceso subjetivo, solamente al agente cognoscente (el otro) cabe la prerrogativa de reconocerme (el yo). El otro posibilita el enriquecimiento de la noción de persona que el yo tiene en la medida que la referencia identificante-reflexiva presentada por aquel no retira o anula, ni siquiera parcialmente, la dimensión existencial de éste, pero simplemente presenta un otro referencial lógico a partir del cual el yo puede ser pensado (Ricoeur, 1990, p. 54).

Pero lo más significativo es que la descubierta de la identidad a través del reconocimiento mutuo convierte aquella idea inicial de exclusión que existía entre el yo y el otro, donde aquel era la negación de éste y viceversa, para un status fundamental en que la existencia del otro $-\mathrm{y}$ la forma como este se manifiesta en el mundo de la vida- afecta directa o indirectamente el existir e incluso la esencia de mi yo (Ricoeur, 2003, p. 241). Es a través del reconocimiento mutuo que se hace posible alcanzar los momentos definitivos del reconocimiento de sí mismo (Ricoeur, 2003, p. 294).

Además de eso, el momento definitivo del reconocimiento será el momento de la determinación de la alteridad en la relación: además de que mi yo se reconozca en el otro, puedo, con base en la alteridad, guiar mi acción como si yo fuera el otro, como si yo estuviera en su lugar, pensando a partir da su noción de bien, la cual es, en ese instante, compartida conmigo (Ricoeur, 1990, pp. 367-369). La alteridad es la constatación de que la constitución ontológica del yo (la ipséité) cuenta con el otro como parte de sí mismo, pues la relación dialéctica entre ambos atesta no la existencia de dos entidades éticas distintas, sino que dos seres autónomos tienen una comunión ética, la cual, aunque sea contingente, tiene en la identidad producida por el reconocimiento mutuo el medio herme- néutico capaz de atribuir una estabilidad reflexiva a la relación ontológica entre ambos.

$\mathrm{Al}$ analizar la temática del reconocimiento en el pensamiento hegeliano, Axel Honneth refiere, en su opus magnum, que el reconocimiento mutuo se desarrolla a través de una sucesión de relaciones específicas en tres áreas distintas: el amor, el derecho y la eticidad, las cuales posibilitan a los individuos confirmarse mutuamente como personas autónomas e individualizadas (2000, pp. 83-84). Honneth habla también de "solidaridad" para designar una forma de reconocimiento de naturaleza superior, pues ella está en condiciones de envolver otras dos formas de reconocimiento: en elle-même [la solidariedad], cette relation se présente comme une synthèse des deux modes de reconnaissance précédents, parce qu'elle partage avec le 'droit' la vision cognitive de l'égalité universelle, avec l' amour' la dimension affective de l'attachementet dela sollicitude(2000, p. 110).

El amor es la forma primaria de reconocimiento, donde el individuo empieza a crear nociones de amistad, confianza en si mismo y afecto hacia el otro con quien se identifica en la forma más íntima posible (Bauman, 2003). Teniendo en cuenta que el reconocimiento es un elemento constitutivo del amor, la relación no se restringirá solamente al plano cognitivo, donde uno considera el otro por su mera existencia; va a definirse a partir de una intersubjetividad que tiene en la afección la causa de la aceptación e identidad recíprocas entre las partes implicadas (Honneth, 2000, pp. 131-132).

Ya el derecho será una dimensión que se establece cuando existe una perspectiva normativa impersonal e imparcial suficientemente capaz de hacer que los miembros de la sociedad se reconozcan como portadores de derechos, es decir, como personas con prerrogativas jurídicas que solamente las pueden ejercer en el ámbito social (Honneth, 2000, p. 132). Al derecho le competirá la tarea de dar "reconocimientoatestación" a los actos que el individuo realice a lo largo de su vida social, civil y política. 
La eticidad es la tercera dimensión de desarrollo del reconocimiento mutuo. Se trata de la "estima social", es decir, de la "honra social" que el individuo busca conquistar. No se refiere a algo vinculado a cualquier relación jurídica y tampoco se limita a las cualidades morales de la persona. Nociones como "prestigio", "respeto" y "consideración" determinan el grado de reconocimiento social que el sujeto busca atribuirse mediante la forma por la cual hace coincidir la realización de sí mismo con los fines abstractamente definidos por la sociedad (Honneth, 2000, pp. 153-154).

La identificación de su conducta individual y de sus valores personales con aquellos definidos por la sociedad de la cual hace parte torna el reconocimiento social en una forma de reconocimiento que evalúa no el individuo, sino sus acciones. Por eso, la capacidad de un grupo en reconocer sus miembros será directamente proporcional al modelo de conducta que la sociedad formada por el grupo estableció, posibilitando que tales requisitos faciliten o dificulten la absorción de nuevos miembros, además de la manutención de los ya presentes. El derecho será formado en un momento subsiguiente al de la eticidad y tendrá como finalidad, sobre todo, dar estabilidad institucional al sistema y atribuir reconocimiento-atestación a los actos producidos por los individuos.

Parafraseando a Honneth, el reconocimiento es el "corazón del social" (2006, p. 154). Por esa razón la doctrina de los derechos humanos, justificada racionalmente por diversas corrientes iusfilosóficas de matriz kantiana -y su inexorable orientación teológica judaico-cristiana- no considera que la noción de reconocimiento tenga una posición central en los sistemas sociales $y$, por consecuencia, en la definición de lo que es ser humano. Además, toda tradición kantiana se funda en la búsqueda de criterios normativos racionales universalmente válidos, dejando en segundo plano -o incluso en el olvido- todos los elementos empíricos que se encuentran en el "corazón" del cuerpo social, los cuales son los responsables por definir y atribuir identidad a individuos, culturas y pueblos.

\section{EL RECONOCIMIENTO COMO PRESUPUESTO DISCURSIVO DE LOS DERECHOS HUMANOS: LA COMPLEJIDAD DE LAS DIVERSAS INSTANCIAS DE NORMATIVIDAD JURÍDICA Y LAS ESPECIES DE RECONOCIMIENTO}

Hasta ahora podemos sacar una conclusión preliminar en el presente artículo: la doctrina de los derechos humanos tiene como presupuestos epistemológicos categorías deontológicas que se caracterizan por la naturaleza descriptivo-prescriptiva de sus postulados normativos. Al mismo tiempo en que describen la naturaleza humana también prescriben conductas inmediatamente decurrentes de tales descripciones. Se puede ver que el objeto descrito (el ser humano) representa el ser que en sí que mueve todo un universo de deber ser que pasa a gravitar y a desarrollarse a su alrededor.

La centralidad axiológica del ser humano dentro de sus propios sistemas sociales no parece generar mayores complicaciones -cualquier ser dotado aunque sea de la más simple racionalidad pone el propio bien y seguridad como marcos referenciales para sus juicios prácticos. El problema está en limitar toda la dimensión discursiva del mundo de la vida a lo que ya fue apriorísticamente establecido por una especie de "consenso universal", algo que, de hecho, no pasa del producto de un estadio evolutivo de una tradición cultural específica, es decir, la tradición occidental de matriz judaico-cristiana, la cual solamente se elevó al estadio actual de desarrollo por haber superado ${ }^{5}$ en su historia violencias y atrocidades no menos crueles que los actos de otras tradiciones culturales que actualmente son repudiados y vistos como contrarios a la humanidad. Por tanto,

\footnotetext{
Se debe entender "incorporado" en el sentido de "incorporado y conservado" de la expresión aufhebegen que Hegel utilizaba para referir la historicidad que el ser posee, de modo que, incluso, la negación de la afirmación está en condiciones de influenciar esta última dentro del proceso de formación de la síntesis, restando "incorporada y conservada" dentro de la síntesis que haya reafirmado aquella afirmación inicialmente negada. Sobre la lógica hegeliana, ver Hegel (1977 y 1972).
} 
lo que proponemos aquí es reflexionar sobre la posibilidad de presupuestos epistemológicos discursivos para los derechos humanos, en vez de presupuestos ético-normativos.

Establecer un espacio comunicacional de construcción normativa procedimentalmente guiado por el reconocimiento-reciprocidad, como ocurre con la actual concepción de derechos humanos vigente, significa que todos los agentes racionales implicados:

(1) son capaces de reconocerse mutuamente, en el sentido de reconocimiento-identificación, o sea, encontrando en el otro la proyección de caracteres subjetivos del yo;

(2) vivencian una misma dimensión cognitiva, de modo que todos puedan ser sometidos a experiencias no limitadas a un único grupo o individuo (por ejemplo, todos viven en una comunidad donde existe o no libertad de expresión; lo que importa realmente es que todos tengan sus existencias sujetas a las mismas variantes fenoménicas);

(3) la comunicación entre los individuos está condicionada por principios racionales comunes que determinan como la construcción del discurso público debe ocurrir, no significando necesariamente que el contenido del discurso ya esté predeterminado; $y$

(4) la continua evolución del proceso comunicacional produce - $y$ afirma- una identidad cultural agregadora de las diversas identidades individuales implicadas, permitiendo que los individuos vean reflejados en aquella determinada comunidad ética caracteres propios de su yo y que son comunes a los demás miembros de esa comunidad.

En suma, el reconocimiento-reciprocidad es una especie de reconocimiento en condiciones de desarrollarse solamente en contextos sociales en los cuales todos los agentes racionales están subjetivamente muy cercanos, como ocurre actualmente con subculturas y microculturas, tornándose inapropiado para la construcción de un diálogo intercultural.
Es oportuno recordar a Habermas cuando sostiene que su concepción elemental de raison communicationnelle s'affirme dans la force de la cohésion inhérente à l'entente intersubjective et à la reconnaissance réciproque; elle circonscrit par là même l'univers d'une forme de vie communautaire (1988, p. 383). La progresiva diferenciación funcional de la sociedad posmoderna, produciendo incontables esferas sociales $e$ instancias normativas, impide que se pueda pensar en cualquier suerte de aproximación bien sucedida, en una misma forma de vida comunitaria, entre individuos que no se reconocen mutuamente y que tampoco se ponen de acuerdo con los primeros principios del discurso racional.

La complejidad de las relaciones sociales ha impuesto al fenómeno normativo la necesidad de diferenciación también en lo que se refiere a las especies de reconocimiento empleadas en las relaciones in concreto. El Estado, que al inicio de la Modernidad tenía la pretensión de ser la síntesis de las identidades individuales, representando el cuerpo colectivo como un todo y, con base en eso, teniendo legitimidad para asegurar la homogeneidad cultural de su sociedad, hoy ya no es reconocido por sus ciudadanos que mediante el reconocimiento-atestación, pues el individuo busca el Estado solamente cuando desea ver una situación dada unipersonal suya de ser atestada o asegurada con efectos contra todos (véase que el reconocimiento-atestación es el instrumento subjetivo legitimador, desde un pedido de emisión de documento de identidad o pasaporte hasta el reconocimiento judicial de un pretenso derecho subjetivo del individuo frente a terceros).

Si por un lado, el reconocimiento-identidad no se presenta en condiciones de generar la aproximación intercultural en los términos que las versiones universalistas de la doctrina de los derechos humanos desearían, por otro, su alternativa inmediata, es decir, el reconocimiento-reconciliación, termina siendo todavía menos adecuada a tal fin. Si la primera requiere que exista una relación entre las partes 
suficientemente sólida para que sea posible desarrollar la identidad ya existente entre las partes, lo que parece ser impracticable cuando pensamos en tradiciones culturales en conflicto incapaces de reconocer la legitimidad de la existencia del otro, ya en el caso del reconocimiento-reconciliación la relación intercultural se caracteriza por la superación de un equívoco en el conocimiento entre las partes que impedía que la relación entre ellas pudiera ocurrir con un mínimo de reconocimiento mutuo.

El objetivo final del reconocimiento-reconciliación es hacer que la superación de las diferencias entre las partes termine por crear una nueva realidad cognitiva en que cada uno de los implicados anteriormente en el conflicto para pensar su identidad individual como correspondiente a la identidad individual del otro. Además de compartir destinos en común, el reconocimiento-reconciliación se basa también en la definición de una pauta deontológica a ser seguida por las partes, las cuales solamente conseguirán eso si son agentes virtuosos.

Sin embargo, en las relaciones interculturales, caracterizadas por la fragmentación de las nociones de valor y de bien, resulta imposible que cualquiera de esos dos modelos de reconocimiento pueda tener éxito. Parece más razonable pensar en tratar de la coexistencia intercultural de partes que no poseen reconocimiento mutuo a partir de un principio de tolerancia válido tanto para una sociedad de ángeles como de demonios, es decir, un sistema que promueva la coexistencia sans faire appel à la vertu morale (Zarka, 2007, p. 30). En ese sentido, Yves-Charles Zarka afirma que el reconocimiento dans un monde déchiré ne peut pas être réconciliatrice, car elle supposerait alors que deux cultures différentes dépassent leurs différences en une autre culture qui en serait plus ou moins la synthèse (Zarka, 2007, p. 40).

Las diferencias culturales, étnicas y teológicas entre tradiciones culturales requieren que se afirme la noción de reconnaissance sans réconciliation. Esta forma de reconocimien- to es, al mismo tiempo, en relación a aquél con quien tengo identidad y en relación al diferente, al extraño, al otro por quien no tengo ninguna identidad. El reconocimiento sin reconciliación posee un contenido mínimo: elle porte sur l'existence et donc sur le droit d'exister. Elle est reconnaissance de la légitimité de l'existence de l'autre culture, de l'autre communauté ou de l'autre peuple (Zarka, 2007, p. 40). Esta modalidad de reconocimiento, implementada en el ámbito de las relaciones interculturales entre tradiciones excluyentes, asegura el derecho a la existencia cultural, independientemente de qué existencia sea. Traspasadas las fronteras de la esfera intracomunitaria, la noción de reconocimiento mutuo se torna inviable o utópica.

Pensar los derechos humanos con base en un principio discursivo determinado por nociones específicas de reconocimiento no significa rever por completo toda la historia de esa categoría de derechos. Como vimos anteriormente, cuando existe identidad cultural entre los sujetos implicados, el problema de la legitimidad de los derechos humanos no se hace primordial, pues fue una misma tradición cultural que construyó aquella idea específica de derecho visto como universal y válido para todo el género humano. La dificultad casi insuperable ocurre cuando se está delante de un derecho, como la libertad de expresión, por ejemplo, que no es compartido por diferentes tradiciones culturales.

En esos casos, el discurso racional necesita estar establecido, fundamentalmente, a partir del reconocimiento sobre la existencia del otro, so pena de restar solamente la guerra y la búsqueda por la aniquilación del otro como alternativas para tal relación. Reconocer la legitimidad de la existencia del diferente debe ser el primer punto de partida discusivo. La afirmación posterior, paulatina y creciente de categorías normativas en común dependerá de que la propia relación intercultural esté en condiciones de desarrollarse sin que sea necesario presuponer la sumisión de una tradición a otra - como pretenden las versiones universalistas de los derechos humanos. 
Ya no siendo posible al Estado moderno asegurar la homogeneidad cultural, las relaciones sociales, económicas, políticas y, por consiguiente, jurídicas, han entrado en un veloz y creciente proceso de diferenciación funcional. Nuevas instancias y esferas normativas surgen y se desarrollan en los más diversos contextos sociales. Ese fenómeno fue incluso llamado de dissolution du lien socia" por Jean-François Lyotard (1979, p. 31). Pero entendemos que el espacio social se tornó, en efecto, en una gran red fragmentada en incontables estructuras sociales que se conectan unas a las otras por tantas otras instancias de normatividad jurídica o moral. Tal disolución que caracteriza las sociedades posmodernas no pasa de una fragmentación de un antiguo conjunto homogéneo en subestructuras transversalmente cortadas por instancias de regulación social.

\section{CONSIDERACIONES FINALES}

El Derecho, en cuanto fenómeno normativo, y el Estado, como centralizador del fenómeno político, sigue siendo insuficiente el tradicional rol de modalidades de reconocimiento, pues, cuando no es posible al Estado asegurar coercitivamente sus acciones, cabe apenas a la aceptación intersubjetiva por parte de sus ciudadanos dar efectividad a determinada acción. En síntesis, la legitimidad del poder político carece de nuevas formas de reconocimiento que estén en condiciones de incluir los particularismos de las muchas realidades factuales implicadas por el Estado.

El reconocimiento mutuo, es decir, el reconocimiento-reciprocidad, permanece aún como instrumento intersubjetivo en mejores condiciones de promover/profundizar el sentimiento de identidad y pertenencia nacional, pues envuelve individuos que ya conducen sus conductas con base en los mismos principios de la razón práctica. Las insuficiencias del reconocimiento-reciprocidad emergen cuando la diferenciación intercultural aumenta internamente $y$ cuando formaciones identitarias externas buscan el reconocimiento político de sus derechos culturales.

Tanto en situaciones en el ámbito nacional como en el ámbito internacional, concluimos que es necesario pensar en una forma de vinculación intersubjetiva basada no en los caracteres esenciales de una tradición cultural específica: debemos reconocer la simples existencia del otro como una situación a legitimar -no en sentido amplio- la afirmación de sus derechos. En otra palabras, eso significa promover un reconocimiento sin reconciliación que empiece el discurso público sin imponer una cultura sobre otra, pero estableciendo puntos de acuerdo y también de desacuerdo intercultural que deberán demarcar los confines del discurso, y principalmente donde se puede llegar, en cuanto un espacio público de coexistencia intercultural.

Dicho eso, vemos que a la doctrina de los derechos humanos, en cuanto fenómeno normativo con pretensiones de universalidad frente a todos los Estados nacionales existentes, resta encontrar o desarrollar modalidades de reconocimiento que estén en condiciones de promover el perfeccionamiento de un espacio público global fundado primordialmente por principios discursivos. Sin embargo, , será necesario abrir pretensiones salvacioncitas y catequizantes en relación a las culturas no occidentales.

\section{REFERENCIAS}

Arendt, H. (2002). A Condição Humana. Rio de Janeiro: Forense.

Arendt, H. (1972). Entre o Passado e o Futuro. São Paulo: Perspectiva.

Bauman, Z. (2003). Liquid Love. On the Frailty of Human Bonds. Cambridge: Polity Press.

Benoist, A. de. (2005). Identità e comunità . Napoli: Guida Editore.

Bobbio, N. (1997). L'età dei diritti. Torino: Einaudi. 
Falk, R. (1999). Predatory Globalization. Cambridge: Polity Press.

Falk, R. (1995). On Human Governance. Towards a New Global Politics. Cambridge: Polity Press.

Falk, R. (1981). Human Rights and State Sovereignty. New York: Holmes and Meier.

Ferry, J. M. (2001). De la civilization. París: Cerf.

Ferry, J. M. (1991). Les puissances de l'expérience. Essai sur l'identité contemporaine. París: Cerf.

Hall, E. T. (1979). Au-delà de la culture. París: Seuil.

Hegel, G. W. F. (1972a). Fenomenologia dello spirito. Milano: Vita e Pensiero.

Hegel, G. W. F. (1972b). Science de la lógique. París: Aubier-Montaigne.

Honneth, A. (2000) La lutte pour la reconnaissance. París: Cerf.

Honneth, A. (2007) La réification. Petit traité de Théorie critique. París: Gallimard.

Honneth, A. (2006). La société du mépris. Vers une nouvelle Théorie critique. Édition établie par Olivier Voirol. París: Éditions La Découverte.

Kant, I. (2003). Fundamentação da Metafísica dos Costumes. São Paulo: Martin Claret.

Lyotard, J-F. (1979). La condition postmodern. París: Les Éditions de Minuit.

Ricoeur, P. (2003). Parcours de la reconnaissance. París: Gallimard.

Ricoeur, P. (1990). Soi-même comme un autre. París: Seuil.
Ricoeur, P. (1967). Histoire e vérité. París: Seuil.

Schmitt, C. (1972). Le categorie del politico. Bologna: il Mulino.

Strauss, L. (1986). Droit naturel et histoire. París: Flammarion.

Taylor, C. (2005). La politica del riconoscimento. En Taylor, C. \& Habermas, J. Multiculturalismo. Lotte per il riconoscimento. Milano: Feltrinelli.

Teixeira, A. V. (2008). Globalização, soberania relativizada e desconstitucionalização do direito. En Teixeira, A. V. \& Longo, L. A. (Eds.). A Constitucionalização do Direito (p. 31-50). Porto Alegre: SAFE.

Teixeira, A. V. (2011). Teoria Pluriversalista do Direito Internacional. São Paulo: WMF Martins Fontes.

Teixeira, A. V. (2009). Global War: the Concept of Modern War under Attack. Mexican Law Review, 2, 2, 89-106.

Teixeira, A. V. (2008). Aporias do Global Constitutionalism de Richard Falk. Revista Direitos Fundamentais \& Justiça, 3, 201214.

Torodov, T. (1989). Nous et les autres. La refléxion française sur la diversité humaine. París: Seuil.

Zarka, Y. C. (2007). Difficile tolérance. París: PUF.

Zarka, Y. C. et al. (2002). Les fondements philosophiques de la tolérance. 3 Vols. París: PUF.

Zarka, Y. C. (2000). L'autre voie de la subjectivité. París: Beauchesne. 